dialectica Vol. 59, № 2 (2005), pp. 237-264

\title{
The Caesar Problem in its Historical Context: Mathematical Background
}

\author{
Jamie TAPPENDEN ${ }^{\dagger}$
}

\begin{abstract}
The issues surrounding the Caesar problem are assumed to be inert as far as ongoing mathematics is concerned. This paper aims to correct this impression by spelling out the ways that, in their historical context, Frege's remarks would have had considerable resonance with work that other mathematicians such as Riemann and Dedekind were doing. The search for presentation-independent characterizations of objects and global definitions was seen as bound up with fundamental methodological questions in complex analysis and number theory.
\end{abstract}

In this paper I'll describe some of the work done in Frege's mathematical environment (in the areas of mathematics he worked, in the styles he did his work in) forming the background against which we can see the Caesar problem. Because we have underestimated what a rich field mathematics was at the time, we've underestimated how natural - to many mathematicians of the time - some of Frege's concerns would seem (though often he pushed them a step or two farther than the people in his environment). In particular, there is a family of issues appearing in Grundlagen in ways that connect to the Caesar problem - the emphasis on global definitions rather than partial ones, defining objects in a representation-independent way, the importance of being able to identify objects in diverse ways and the connection of that to extending knowledge, the general emphasis on the importance of fruitful concepts - that would have reverberated in very specific ways with Frege's mathematical readers. Furthermore, the themes that would have echoed for Frege's mathematical readers were so salient that it is highly unlikely that in writing Grundlagen, Frege wasn't aware of the allusions that his mathematically informed readers could be expected to infer. This will help bring out that even in what appear to be Frege's most 'philosophical' moments, there is far more going on than meets the eye. In fact, there is so much going on behind the curtain that I am not going to cover all aspects of the mathematical environment that are relevant to some aspect of Frege's treatment of the Caesar problem. I'll spell out enough of the background to illustrate how it can bring out hidden riches in a couple of aspects of the Caesar problem; that should be enough for a start.

\footnotetext{
Department of Philosophy, 2228 Angell Hall, University of Michigan, Ann Arbor MI 48109; Email: tappen@umich.edu
} 
There is a widespread view, which has even been defended in print, that Frege's foundational work is mathematically inert: that it is disengaged from the mathematics around him and is not in the same spirit. Certainly this is taken to be true of his concern for explicitly presenting numbers as objects, so that the definition supports the conclusion that Julius Caesar is not a number. It is not we are inclined to suppose - the sort of thing that mathematicians regard as useful; and to be sure it has not proven to be of much mathematical use. This point is not just a contemporary novelty: we seem to find it in the opinion of the young Husserl, fresh from his Weierstrass-inspired $\mathrm{PhD}$ on the calculus of variations, who dismissed the 'fruitless subtlety' of Frege's Grundlagen. ${ }^{1}$ And certainly if we are to judge by his non-foundational work, Frege was a decent but certainly not a great 'mainstream' mathematician. ${ }^{2} \mathrm{He}$ was astute at analyzing and laying out the logical structure of innovations in mainstream practice that others had demonstrated to be rich and important, but he was not especially gifted at producing or applying such innovations himself. ${ }^{3}$

But in contrast to our contemporary preconceptions, Frege thought he was doing something that would have an impact on non-foundational practice. In his motivating remarks at the outset of Grundlagen he writes of his 'further hope' that logically clarifying the fine structure of the simple examples will allow the clarified concepts to be carried over to more intricate questions.

The aim of proof is, in fact, not merely to place the truth of a proposition beyond all doubt, but also to afford us insight into the dependence of truths upon one another. . . . But there may even be reason for a further hope: if, by examining the simplest cases, we can bring to light what mankind has there done by instinct, and can extract from such procedures what is universally valid in them, may we not thus had in mind.

${ }^{1}$ Husserl 1891. Of course, it might have been fruitlessness for philosophy that Husserl

${ }^{2}$ By 'mainstream' or 'non-foundational' mathematics I mean to refer to Frege's extensive work in those areas of mathematics outside logic and foundations, principally complex analysis and geometry. I am using the label only for orientation, and I assume that readers will recognize what I am getting at; I do not mean to suggest that the distinction is a deep or principled one.

${ }^{3}$ A lovely paper (Gronau 1997) has convinced me that this observation needs one qualification: though Frege did not make any real mark on nonfoundational mathematics, his Habilitationsschrift apparently shows a talent for unearthing novelties that were, in hindsight, quite promising. (The importance of these novelties was rediscovered and developed by others much later, not by Frege.) Gronau suggests plausibly that much of [MCE] is potentially quite important, though Frege never returned to the iteration-theoretic dimensions of this work. (Though nearly all that I know about iteration theory is what I learned from Gronau's paper, so I am no position to give an expert assessment.)

There is, in fact, a distant affinity between the iterative methods Frege studied and a technique introduced by his erstwhile student Heinrich Liebmann in Liebmann 1918, for solving families of partial differential equations. This may represent one of the rare points at which the influence of Frege's non-foundational work showed itself in subsequent mathematics. 
arrive at general methods for forming concepts and establishing principles which will be applicable also in more complicated cases? ([FA] 2; see References for key to bracketed Frege references in text).

Frege says that clarifying simple and basic cases might illuminate more complex ones. This 'further hope' seems to be that through foundational analysis of elementary cases like the concept of number, problem-solving in more complicated contexts will be facilitated. That is, Frege marks out as one of his objectives that he aims to facilitate problem-solving in general mathematical practice. ${ }^{4}$ This is echoed elsewhere, when he discusses the criterion for judging a concept especially worth logical attention:

All these concepts have been developed in science and have proved their fruitfulness. For this reason what we may discover in them has a far higher claim on our attention than anything that our everyday trains of thought might offer. For fruitfulness is the acid test of concepts, and the scientific workshop is logic's true field of observation ([BLC] 33).

The 'further hope' was not unreasonable in principle. Facilitating results at the frontiers of nonfoundational mathematics by clarifying the foundations of simple concepts did happen at the time. To consider just one example we'll revisit in section 3, in work that Frege was sure to have been familiar with at least in outline, Dedekind and Weber 1882 (two years before Grundlagen) reconstructed Riemann's theory of algebraic functions of one variable in what we would now call algebraic terms. This was an astonishing triumph of logical analysis (broadly understood) since it had as one key the fine structure of factorization revealed by Dedekind's generalized notion of division among numbers, carried over to the more intricate case of algebraic functions. This generalized notion of division had at its core Dedekind's treatment of ideals, which we'll consider in section 3 . This was a triumph in other ways that matter to Frege as well. In particular, it exploited the refined concepts of number and division to rework, without any appeal to geometric intuition, concepts and theorems at the 'cutting edge' of ongoing research that a reasonable person could have thought at the time were essentially geometric, relying crucially on intuition.

Frege was working during a time that was especially exciting in mathematics, during which many of the basic assumptions about the nature of the discipline were reoriented. With the passing of time, we have lost the ability to naturally

\footnotetext{
${ }^{4}$ That the 'more complicated cases' are expected to occur in mathematics is conveyed by the context of the quote. In the section in which it occurs, §2, Frege is explicitly discussing what he classes as mathematical motives in section $\S 1$. He begins the next section by drawing an explicit contrast between philosophical motives and what went before: 'Philosophical motives too have prompted me to inquiries of this kind' ([FA] 3) (He makes this distinction of motives also in the annotated table of contents.) That Frege explicitly marks out this distinction in his motives at this point was, I believe, first pointed out in Benacerraf 1981.
} 
pick up on allusions and borrowed clichés that would have been evident to his readers. As we'll see, many notes that are played in Grundlagen would have resonated with his mathematical readers in predictable ways, and Frege should be expected to have written with an awareness of how his words would strike his audience - or at least the mathematically informed among them. In particular, we'll see that there are surprisingly many hidden dimensions to Frege's words. Among the charged topics were Frege's emphasis on fruitfulness, his search for global definitions, and his search for the presentation - independent introduction of an object in a way that permits a multifaceted approach to it, with the suggestion that such presentations have a connection to objectivity and extensions of knowledge. I should warn the reader in advance that the balance between discussion of specific Fregean passages and details of the mathematical background tilts to the latter. I will not discuss all of the aspects of the Caesar problem; indeed I won't even discuss all those aspects of Frege's discussion of the Caesar problem that have parallels in Frege's mathematical environment. I'll just take up a few passages and dialectical moves, to illustrate how much currently forgotten information and background would have brought to life even single turns of phrase for Frege's readers.

To help make plausible the suggestion that the mathematical background influenced Frege as I'm suggesting it did, I'll concentrate on the way that Dedekind was moved to carry out a maneuver similar to the one Frege exhibits in response to the Caesar problem. This strategy should not be taken as suggesting that Frege and Dedekind were alike in all respects: clearly there are important differences. Rather the point is to combat an entrenched stereotype. Despite what the context may suggest about how to read various passages where Frege writes in a way that any mathematician of the time would regard as highly charged, there might be resistance based on an armchair sense that mathematicians simply don't think that way about the relation between foundations and ongoing practice. Dedekind's words and practice will undercut that resistance, by showing that at that time, one mathematician with affinities to Frege not only thought that way, but said so explicitly and acted on those thoughts in detail to contribute to revolutionary nonfoundational work.

\section{Definition-independence of the presentation of objects and the fruitfulness of concepts}

Even those who have thought long and hard about the Caesar problem can find themselves with occasional nagging doubts as to just what the problem is, and why it should have the importance to Frege that it does. Even when we figure out what the problem is supposed to be, many of the things Frege says about it don't seem to ring just right. How does this have anything to do with any idea of 
'self-subsistence' which objects are taken to have? How do Frege's remarks to the effect that an independent mode of presentation is needed to secure more than unfruitful inferences fit in?

A clear statement of the connection among some of the ideas in the cluster we'll explore occurs at Grundlagen $\$ 67$. The remarks come in response to a natural question that might be asked in connection to the Caesar objection: why not say that the things whose introductions are explicitly forced by the definition count as numbers (or in this case directions), and nothing else does? Frege's response ties the definition-independence of the presentation of objects to the potential for increasing knowledge through deductive reasoning.

\$67 If we were to try saying: $q$ is a direction if it is introduced by means of the definition set out above, then we should be treating the way in which the object $q$ is introduced as a property of $q$, which it is not... [and a further unacceptable consequence would be that:] All identities would then amount simply to this, that whatever is given to us in the same way is to be reckoned as the same. This, however, is a principle so obvious and so unfruitful as not to be worth stating. We could not, in fact, draw from it any conclusion which was not the same as one of our premises. Why is it, after all, that we are able to make use of identities with such significant results in such diverse fields? Surely it is rather because we are able to recognize something as the same again even though it is given in a different way ([FA] 78-79 emphasis mine).

We should recall that although today mathematics has advanced to the point that the introduction of objects with reference to some equivalence relation is old hat, to Frege it was a relatively novel device for defining direction (orientation, etc.); Frege would have seen himself as exploring a new technique whose potential wasn't fully charted. Outside of geometry a high profile use of a cognate maneuver was exploited in Dedekind's reconstruction of Kummer's theory of ideal numbers. We'll consider that, and the motivations for it, in 3). First let's remind ourselves of how Frege regards the connection between his conception of fruitful mathematical innovation and the Grundlagen conception of 'extending knowledge'. Frege remarks in Grundlagen $\$ 64$ that in the Hume principle 'we carve up the content in a new way and this yields us a new concept (75).' These metaphors of carving are marshaled (elsewhere in Grundlagen as well as in other Fregean writings) to support the suggestion that logical inferences exploiting the truly 'fruitful definitions' (sometimes 'fruitful concepts') actually extend knowledge. In Frege's most vivid expression of the point, he writes:

[Kant] seems to think of concepts as defined by giving a simple list of characteristics in no special order; but of all ways of forming concepts, that is one of the least fruitful. If we look through the definitions given in the course of this book, we shall scarcely find one that is of this description. The same is true of the really fruitful definitions in mathematics, such as that of the continuity of a function. What we find in these is not a simple list of characteristics; every element is intimately, I might almost say organically, connected with the others ([FA] 100). 
Frege has other remarks scattered around his writings in which he indicates further the kind of advantages that he takes to be involved in the definitions he calls 'fruitful'. In characterizing his reasons for pushing more deeply than usual into logical foundations, he notes a cluster of desiderata for definitions that animate mathematical practice, including '[revealing] connections between matters apparently remote from one another' and 'leading to an advance in order and regularity'. Though he regards these as insufficient unless supplemented by logical foundations, he takes them to tend in a general way toward getting things right:

If a definition shows itself tractable when used in proofs, if no contradictions are anywhere encountered, and if connections are revealed between matters apparently remote from one another, this leading to an advance in order and regularity, it is usual to regard the definition as sufficiently established, and few questions are asked as to its logical justification. This procedure has at least the advantage that it makes it difficult to miss the mark altogether. I agree that definitions must show their worth by their fruitfulness: it must be possible to use them for constructing proofs.... [Though by] these methods we shall, at bottom, never have achieved more than an empirical certainty ... (Grundlagen, ix).

There are two sides to Frege's conception of fruitfulness of concepts that we should note. First, there is his informal and pre-theoretic view that some concepts and definitions (and, by extension, some theories and frameworks) better facilitate important new discoveries. This is, of course, hardly a controversial view, but what gives it bite here is the second aspect: Frege regards it as an advantage of his system, and describes it as a constraint on an adequate foundation of mathematics, that it should support a representation of 'the truly fruitful concepts'. This is implicit in his discussion of extending knowledge in the Grundlagen, but it is most explicit in the unpublished essay [BLC]. In particular, as noted above, in that essay Frege emphasizes that he regards his foundational effort as bound to address the concepts that are 'most fruitful' as revealed in 'scientific workshops: logic's true field of observation'.

The emphasis on logical decomposition in the 'Boole's Logical Calculus ...' paper as a whole fits with the idea of fruitfulness for a particularly compelling dialectical reason. Frege points out that the concepts he can represent and his critic Schröder cannot are ones that mathematics and science really need. Among the particular cases Frege considers are crucial distinctions in analysis (including formulations of the notions of uniform convergence and continuity for functions) plus notions like 'prime factor' and others concepts relating to the divisibility and factorization of natural numbers. In my paper Tappenden 1995a I studied the examples of continuity and convergence, to bring out how they exemplified Frege's analysis. ${ }^{5}$ Here I'll have more to say in connection with factoring and divisibility.

${ }^{5}$ When I wrote Tappenden 1995a my explorations into Frege's mathematical environment were still in their infancy, and though I was concerned in that paper to argue that Frege's 
Here too, Frege's remarks about fruitfulness and scientific importance were being borne out by well-known events contemporaneous with his logical research.

The areas of mathematics where Frege worked (here we'll consider complex analysis) especially in the style in which Frege was immersed (Riemann's) were in a period of remarkable turbulence. Questions of the proper formulations of problems and the proper choice of basic concepts, attempts to find the 'best' proof methods and disputes over the criteria of 'bestness', and other such broadly methodological topics were particularly salient. Not only were these issues implicit in the ongoing research programs of the mathematicians involved: those who were involved in these developments were fully aware, and on occasion quite explicit, about their methodological and (broadly) philosophical preferences. Many other mathematicians in Frege's stream of mathematics also expressed the opinion that something remarkable and quite novel was going on in mathematics which demanded broader methodological clarification. This was in fact a reasonable opinion to express: in the Riemann stream of complex analysis, remarkable things were going on that demanded clarification. Frege's remarks on 'fruitful concepts' were an astute - and not atypical - expression of one side of an ongoing methodological debate. Here I'll present just a slice of this background with relevance to the Caesar problem, but even with the horizon so drastically narrowed we'll see a lot going on that we miss today.

\section{Mathematical context}

To appreciate the relevance of the material of this section to Frege's mathematical environment I'll need to set down a few points that I develop and defend elsewhere. To keep this as a paper rather than a book, I won't defend them here. ${ }^{6}$

b) German complex analysis at the time exhibited a sharp divide between the 'computational' approach of Weierstrass in Berlin, and the 'conceptual' approach of Riemann late of Göttingen.

c) The adherents of the Riemann tradition included Frege's teachers and Dedekind. More generally, the evidence from Frege's teaching, research and context indicates that he was immersed in the Riemann tradition and opposed to the principles driving the Weierstrass approach.

mathematical environment was substantial, I'm now inclined to feel I underestimated the richness of the background. In particular, in connection with the issues of disentangling geometry and analysis, along with the concern about quantifier interaction and continuity-convergence concerns, I would have devoted more time to the Dirichlet principle were I writing that paper now. I rectify that omission in Tappenden forthcoming a and forthcoming $\mathrm{b}$.

${ }^{6}$ See Tappenden forthcoming a and $\mathrm{b}$. 
d) Frege's reading and teaching reveal that the small list of topics Frege concentrated on in his complex analysis research included the theory of elliptic functions (and the generalization to Abelian functions, though we will not consider that here) and the theory of cyclotomic extensions ('circle division'). These two topics were in fact bound together in a delicate way. These topics put stress on the issue of disentangling geometry and arithmetic. Much of Dedekind's most powerful mathematical work was devoted to developing the theories of these, and more general issues in a geometry-free fashion. ${ }^{7}$

A particular feature of Riemann's approach to complex function theory that is relevant here is the way it understood the quest for the 'right' definition of key functions and objects. Dedekind sums up the attitude vividly in the following remarks, in which he represents his approach - moved by Riemann - as pushed forward by an emphasis on 'the internal rather than the external':

[Gauss remarks in the Disquisitiones Arithmeticae]: 'But neither [Waring nor Wilson] was able to prove the theorem, and Waring confessed that the demonstration was made more difficult by the fact that no notation can be devised to express a

${ }^{7}$ In Tappenden 1995a I stressed the importance for Frege of the theme of disentangling geometry and arithmetic, with special reference to the practice of geometry. As I've learned more about Frege's environment, I've learned that the point cuts even deeper with reference to complex analysis. Indeed, a common piece of received wisdom about the nineteenth century mathematics is not only wrong, but drastically so. One statement of the received wisdom occurs in a recent introduction:

The attempt to clarify these notions (of limit, and continuity) involved arithmetizing analysis, that is, showing that its truths could be proved from truths of arithmetic. By the time Frege began his work, most proofs of analysis had been separated from geometry and the notion of magnitude. It is not surprising, then, that it would have seemed less evident to Frege that the truths of analysis are synthetic a priori (Weiner 2000, 19-20).

So far as complex, rather than real, analysis is concerned (this is remember, the kind of analysis Frege worked on) this doesn't stand comparison with the facts. Indeed it completely inverts the historical situation in a way that crucially misrepresents the relation between Frege's foundations and ongoing non-foundational research. Prior to Riemann's work (which drew on Gauss' earlier geometric representation of complex numbers) complex analysis in Germany had been carried out almost exclusively computationally, in the tradition of 'algebraic analysis' with no connection to intuition. It was in the years after Riemann's revolution in the 1850's that complex analysis, in the tradition Frege belonged to, became something different - immersed in intuitive representations. During this period (which we may somewhat arbitrarily terminate with Weyl's rigorous definition of Riemann surface in 1913) it would have seemed less obvious that complex analysis could be carried out independently of appeals to geometric facts than it had to previous generations of mathematicians. In this domain, Weierstrass' 'arithmetization' program - the details of which Frege criticized beginning as early as Grundlagen and continuing through Grundgesetze II - was a parochial and in some respects (such as the attitude to Riemann surfaces) even somewhat hidebound stance for clinging to the old certainties in the face of a completely novel style of mathematics. I discuss this point further in Tappenden forthcoming a and $\mathrm{b}$. 
prime number. But in our opinion truths of this kind ought to be drawn out of notions not out of notations.' In these last words lies, if they are taken in the most general sense, the statement of a great scientific thought: the decision for the internal in contrast to the external. This contrast also recurs in mathematics in almost all areas; [For example] (complex) function theory, and Riemann's definition of functions through internal characteristic qualities, from which the external forms of representation flow with necessity [Dedekind continues, in paraphrase: The contrast also comes up in ideal theory, and so I am trying here to put down a definitive formulation] (Dedekind 1895, 54-55).

Of course, the philosophical question of how to distinguish 'fundamental characteristics' that allow you to 'predict the results of calculation' from 'forms of representation which should be results, not tools, of the theory' is complicated. But in the specific cases at hand, particularly in Dedekind's favored analogue, Riemann's complex function theory, the cash value of the contrast - in terms of known examples - is easily established and it would have been transparent to his readers what he was referring to. The point of contrast that we're particularly concerned with is: are objects of study to be introduced in a way that ties them to a specific representation, or in a representation-independent way allowing indirect existence proofs and the discussion of objects for which no description is available? In that case, the choice was one of inestimable mathematical significance.

In section 3 we'll further anchor the point in the philosophical issues familiar to Frege scholars, by considering how Dedekind explicitly links the Riemann methodology and orientation to definitions with his version of the 'Caesar problem', when he reformulates Kummer's ideal theory in a representation-independent way. As we'll see, Dedekind uses just this charged language to motivate the transition from what Frege would call 'a concept' to what he would call 'an object'.

It would be a distraction to compile a longer list of quotations in this vein: suffice it to note (as I spell out elsewhere) that such expressions of opinion - in these words or minor variants - were so common among those in the Riemann stream as to reach the status of clichés. What sorts of reasons were given to justify claims that some definition or other was based 'on fundamental characteristics' instead of 'externals'? It would be disappointing if the only justification Riemann, Dedekind et al. could provide for such a claim were to be some kind of brute, inarticulate and unarticulatable aesthetic response, as if 'being the right definition' were grounded in nothing more than 'powerfully seeming to be the right definition'. In fact, the reasons were subtle and various. I'll concentrate on just one here: it was seen as evidence that a definition got things right if it was fruitful.

Riemann used a particular turn of phrase to make this point: he described the definitions Dedekind is referring to as making it possible to see 'practically without computing' (fast ohne Rechnung) results which had required 'tiresome computations' (mühsame Rechnungen). It was seen as a strength that by present- 
ing objects as Riemann did, a wide range of different perspectives became available. This sort of talk also became clichéd; to consider one example, even as late as 1899 the author of a textbook on elliptic functions (based on Riemann's earlier lectures) characterizes the one strength of the Riemann approach as lying in:

... the synthetic treatment of analytic problems which builds up the expression for the functions and integrals solely on the basis of their characteristic properties and nearly without computing from the given element and thereby guarantees a multifaceted view into the nature of the problem and the variety of its solutions (Stahl 1899, III emphasis mine).

These improvements in evidentness brought out by a proper definition were expected to be systematic: the proper definition should make a range of interesting problems easier, in the long haul. ${ }^{8}$ It is worth noting that the innovations regarded as fruitful by the people we are discussing tended to be rightly so regarded: it is remarkable how much the basic shape of complex analysis and the relevant domains of algebraic geometry today was laid in place by Riemann. The style of thinking he helped introduce dominates contemporary mathematics.

To get a robust sense of the differences at issue between schools of complex analysis in the nineteenth century, we'll need some examples. One example (the definition of analytic function) is simple but not all that deep, and the other (the definition and study of elliptic function) is quite rich, but complicated enough that I'll only be able to point out a few highlights.

Weierstrass' approach, in tune with his 'arithmetization of analysis', takes as basic the definition of an analytic function centered at $z_{0}$ as a function that can be represented as a complex power series $\mathrm{f}(\mathrm{z})=\Sigma \mathrm{a}_{\mathrm{i}}\left(\mathrm{z}-\mathrm{z}_{0}\right)^{\mathrm{i}}$ where the $\mathrm{a}_{\mathrm{i}}$ are complex numbers. This is an infinite series, but it was important to Weierstrass that the definition exploited only 'elementary' terms (addition, multiplication and (formally defined) exponentiation). An important fact about this definition is that it is intrinsically local: the series will typically converge only within some given radius of convergence. Beyond that the series need not be defined. This is not as much of a handicap as it might seem to be at first sight: in those cases where the analytic function on a given open set extends to a multiple-valued function ('multifunction') on the entire complex plane, this continuation is unique. That is, every analytic function has a unique 'natural' continuation to an analytic multifunction defined more broadly. ${ }^{9}$ The key point for our purposes is that there is a specific scheme of representation built into the definition of analytic function.

${ }^{8}$ I might note in passing the interest of this kind of phenomenon for anyone who wants to rest any weight on the idea of basic principles 'being evident'. 'Being evident' is systematically dependent on the theoretical context and even the definitions of basic core ideas.

${ }^{9}$ Note, though that a 'multifunction' is not a function, as this expression is defined in elementary logic and calculus textbooks, since it assigns several values to one argument. To assign unique values, the domain has to be unfolded into a Riemann surface. 
Riemann's approach differs even in the definition of the object of study: the details are less crucial here so they may be left for a footnote. ${ }^{10}$ The key point is that Riemann's definition did not refer to any explicit representation of a function in terms of anything like a series. It was fundamental to his approach that the existence of a given function could be proven, without a representation being sought.

Despite the fact that the definitions are now known to be in some sense interchangeable, the choice of one or the other as basic was regarded as a matter of no little significance. For those in the Riemann school, it was his definition that really cottoned on to the essential properties; the Weierstrass definition exploited accidental forms of representation. Weierstrass objected to the Riemann definition on the grounds that it rested on a primitive and (Weierstrass thought) uncontrolled concept of function. In retrospect, the idea of function has become so central to working mathematics that it is hard to fathom this reservation. I don't think it unreasonable to say that Riemann was just right about the concept of function, and subsequent mathematical developments have made that plain. ${ }^{11}$ (Of course, Frege's view of the fundamental importance of the concept of function not only for mathematics but for 'conceptual thought' is well-known.) There is more, but this will perhaps be enough to make the point: even with quite simple definitions that were largely interchangeable, deep reasons could be given for singling out one of them as 'fastening on the essential, internal characteristics', and these choices were bound up with deep features of ongoing research.

This was a point of concern for Weierstrass, who expressed misgivings about this and related aspects of the Riemann approach in a letter:

${ }^{10}$ For Riemann, the functions to be studied are those satisfying the so-called CauchyRiemann conditions, which define complex differentiability in terms of (real) partial derivatives:

With $\mathrm{z}$ as the complex variable, and writing the real and imaginary parts of $\mathrm{f}$ as $\mathrm{u}$ and $\mathrm{v}(\operatorname{so} \mathrm{f}(\mathrm{z})=\mathrm{u}+\mathrm{iv}), \mathrm{f}$ is differentiable at $(x, y)$ if these partial derivatives exist and these relations hold:

$$
\frac{\partial \mathrm{u}}{\partial \mathrm{x}}=\frac{\partial \mathrm{v}}{\partial \mathrm{y}} \quad \frac{\partial \mathrm{u}}{\partial \mathrm{y}}=\frac{-\partial \mathrm{v}}{\partial \mathrm{x}}
$$

These definitions are now known to be (with a couple of additional minor hypotheses) equivalent. This was not known to be true at the time. Riemann himself, who was less rigorous than most of the people - Dirichlet, Frege, Dedekind, Weierstrass - that we are discussing, appeared to assume that any function satisfying the C-R equations at a point could be expanded into a power series about that point, and hence that the function was analytic in Weierstrass' sense (since the converse direction is trivial). I'm grateful to Howard Stein for bringing me to appreciate this. However, Weierstrass, as well as Riemann students like Dedekind, were less sanguine about such things.

${ }^{11}$ There is more to say about the role of indirect function existence arguments as a key to Riemann's approach, and the connection to Frege's analysis of analysis, but I will leave the topic for elsewhere. 
At first the purpose of these lectures was to properly determine the concept of analytic dependence; to this there attached itself the problem of obtaining the analytic forms in which functions with definite properties can be represented ... for the representation of a function is most intimately linked with the investigation of its properties, even though it may be interesting and useful to find properties of the function without paying attention to its representation. The ultimate aim is always the representation of a function (Weierstrass 1886/1988, 156, emphasis in original). ${ }^{12}$

The contrast at issue can be nicely illustrated by elliptic functions, so it's time to say what these are. 'Elliptic functions' were originally introduced as the inverse of certain kinds of integral. Later it was discovered that elliptic functions have two periods. That is, if $\Phi$ is an elliptic function, there will be complex numbers $\omega_{1}$ and $\omega_{2}$ such that for any complex $z$, and any integers $m$ and $n, \Phi(z)=\Phi(z+$ $\left.m \omega_{1}+n \omega_{2}\right)$. Later still, it was recognized that this is a reasonable choice as a defining feature of such functions, while the original definition in terms of the inverse of an integral, though equivalent, is better seen as accidental. Reflecting this, most textbooks today define 'elliptic function' as a (meromorphic) complex function with this characteristic property.

To approach individual elliptic functions, there is a characteristically Weierstrassian way to go and many characteristically Riemannian ways, among which one is especially handy for illustration here. The Weierstrassian way comes up with a universal scheme for representing elliptic functions in terms of a specific family of series (Weierstrass $\wp$-functions). The Riemann way observes that when you know a few basic facts about the values of an elliptic functions (the places where it is zero, the places where it is infinite (poles), and (loosely speaking) the number of times each of the zeros and poles are to be counted (the orders of the zeros and poles)) then you've determined the function up to a constant, which from a mathematical point of view is to determine it uniquely. With this result in hand, plus others indicating that if you know about zeros and poles you know much of what you need to know, the Riemannian can take the zeros, poles and their orders to be a determining property of a given elliptic function, with a representation developed later, only if needed. Here again we have an illustration of a fundamental Riemannian methodological stance: rather than take it as given at the outset what the basic concepts should be (in the way that Weierstrass took it as given at the outset that a reduction to 'elementary terms' was a reduction to the traditional standbys like plus and times) Riemann regarded the question of what should count as basic as itself something that was to be discovered in the course of research. One criterion for deciding if the proper basic concepts had been identified was the assumption that the correct choice of basic concepts would

${ }^{12}$ I have taken the translation from Shenitzer's translation in Laugwitz 1999. 
yield fruitful characterizations that allowed one to see 'practically without computing' previously obscure results. ${ }^{13}$

As Dedekind sees it, Riemann showed in his treatment of complex functions that there is a great mathematical advantage to be gained by defining the objects of study in a representation-independent way. Dedekind put this attitude into practice in profound work in function theory; for example in his 1877 treatment of elliptic modular functions he exploits Riemannian methods to powerful effect. He was consciously putting into practice the broader methodological stance. As he puts it in a letter to Lipschitz in $1876:^{14}$

My efforts in number theory have been directed toward basing the work not on arbitrary representations or expressions but on simple foundational concepts and thereby - although the comparison may sound a bit grandiose - to achieve in number theory something analogous to what Riemann achieved in function theory, in which connection I cannot suppress the passing remark that Riemann's principles are not being adhered to in a significant way by most writers - for example even in the newest works on elliptic functions. Almost always they mar the purity of the theory by unnecessarily bringing in forms of representation which should be results, not tools, of the theory (Dedekind 1876a, 468-469).

In the next section we'll see how Dedekind approaches the definitions of objects in number theory in this representation-independent way. There are two more points I need to mention before moving on. First of all, a point that will be too complicated to develop here but which should be mentioned is touched on in the remarks quoted above from Stahl's textbook, to the effect that in Riemann's approach one gains a 'multifaceted view' of the problem. For the sake of space, I've omitted a crucial feature of Riemann's approach to analytic functions. Rather than functions defined on the complex plane they are reinterpreted as functions on the 'natural' surfaces ('Riemann surfaces'). The properties of these surfaces reflect key properties of elliptic functions and generalizations (Abelian functions). One side effect of this approach is the number of different routes by which the theory can be investigated: as a geometrically presented theory of complex functions (Klein etc.), as an algebraic theory of curves and surfaces (Clebsch etc.; in an old-fashioned algorithmic sense of 'algebraic'), as a unified algebraic theory of function fields and number fields capturing the algebraic structure of such surfaces and functions on them (Dedekind, in the contemporary 'structural' sense of 'algebraic'), as what we would now call a topological theory (much contemporary work) and many others. The theory can even be reconstructed within the Weierstrass approach, as Klein and Hermann Weyl (in the early part of the

${ }^{13}$ Here too there is more than I can include in a single paper: in particular the role of topology in Riemann's characterization of elliptic functions illustrates deep points about mathematical method. I discuss this further in Tappenden in preparation c.

${ }^{14}$ This letter was brought to my attention by Edwards 1987, 14. I've also taken the translation from that article. 
twentieth century) were at pains to spell out. There are many other ways that appeared in the twentieth century; I've listed a few of the more prominent approaches that were recognized at the time.

The situation is reminiscent of the well-known developments of about the same time in which arguments for the existence of atoms were based on the fact that several different kinds of phenomena (Avogadro's number, Brownian motion, etc.) all pointed to the existence of atoms. (Of course, since the facts in the mathematical case don't have the contingency of those in the empirical case, the analogy is only partial.) Here there are a range of seemingly drastically different mathematical theories that support the same definitions and results. That this was an important and novel development, characteristic of the stream of mathematical research Frege inhabited, should be borne in mind when reading his remarks about the importance, for extending knowledge, of the possibility of recognizing an object as the same under a different guise. The possibility of learning about mathematical objects through widely varied channels of information also adds a recognizable sense to the idea of logical objects as 'self-subsistent' and autonomous in some sense that resembles the autonomy of physical objects. This kind of autonomy is provided by giving the object about which one can theorize rather than symbols to manipulate.

A final point that needs emphasis is that Riemann's techniques often require defining functions more broadly than might otherwise seem necessary. The point is that he systematically exploited interactions between the properties of a domain 'in the small' and 'in the large', so that even in cases where it would appear that only the local facts about objects in a domain were at issue, there was a need to be clear on the facts about the entire domain as a collective. (In contemporary mathematics local-global interaction is a standard pattern, but at the time it was a Riemannian novelty.) I'll give one example to illustrate the point about local and global interaction; I'll also restrict attention just to how this example illustrates local and global interaction, leaving to another time the exploration of additional ramifications of this example in connection to Frege.

A core result in the development of Riemannian analysis is the Riemann mapping theorem. ${ }^{15}$ First a definition: A conformal mapping is a mapping that preserves oriented angles between lines intersecting at a point. With this definition in hand we can state the theorem:

${ }^{15}$ For a clear, historically sensitive discussion of the theorem from a contemporary perspective a good place to turn is Remmert 1991, ch. 8. Walsh 1973 contains an exceptionally clear discussion, which brings out nicely the connection between the original arguments for the Riemann mapping theorem and the Dirichlet principle. A more rigorous and detailed (also more advanced and difficult) exploration of these connections is in Siegel 1969, ch. 2 especially §6$\S 9$. As with many other topics, I owe a debt here to the work of Gray, in this case his 1994. 
Given any simply connected plane region S (alternately: a plane region $\mathrm{S}$ bounded by a simple closed curve) there is a conformal mapping taking $\mathrm{S}$ onto the interior of the unit circle. (i.e.: the disc $\{z|| z \mid<1\}$ ). ${ }^{16}$

Among the interesting things about this result is that to prove it, Riemann invoked a controversial principle - the 'Dirichlet principle' - whose history also forms an important backdrop to Frege's work. ${ }^{17,18}$ But I'll leave that for elsewhere. Here we should just note that the theorem states a delicate relationship between a global property - i.e. a property of the region as a whole (what we would now call the topology of the region) and a local property (i.e. a property that makes sense in an arbitrarily small neighborhood of a given point): the preservation of directed angles at a point. This is more than just a surprising result: it is, and was recognized as, a harbinger of an entirely new vision of what mathematics can be. (Indeed, much of contemporary mathematics has played out successively more sophisticated variations on this theme.) The key point for our purposes is the observation that the study of apparently narrowly delineated phenomena like angles at a point may require information not just about every other point in the region but even about properties of the collective. This puts pressure toward defining properties more globally than might appear to be required at first sight. I take up another illustrative example - Riemann's treatment of the zeta-function - in the appendix.

\section{Dedekind's 'ideals' and the 'Caesar Problem': (A hidden subtext to Grundlagen)}

So far we've looked at a few related themes from Grundlagen, centering about the Caesar problem, plus some aspects of Frege's mathematical environment that have at least a superficial affinity with some aspects of the Caesar problem. But how close would the affinity have seemed to Frege and his mathematically informed readers? To bring out that the answer is 'very close indeed' it will be

${ }^{16}$ To understand the point it isn't necessary to know what 'simply connected' means. It suffices to know that it is a property of the region as a whole.

${ }^{17}$ The use of the Dirichlet principle to prove the existence of functions required for the Riemann mapping theorem is one of the most salient illustrations of the point mentioned in footnote 7: in complex analysis after 1850 it became unclear to what extent geometry and complex analysis could be untangled. The principle is obvious for simple geometric and physical situations and false in a natural abstract formulation. It was not till the turn of the century that Hilbert came up with a non-geometric version that was both provable and capable of doing the work required of it.

${ }^{18}$ Another point is worth noting (for further details see Tappenden forthcoming b). An expression Frege uses in Grundlagen, to the effect that by providing rigorous proofs one can establish the 'limits to the validity' of a proposition, was also a widely dispersed catchphrase in the late nineteenth century. It showed up in many different areas, but it was especially common in discussions of prospective proofs of the Dirichlet principle. 
helpful to walk through Dedekind's definition of ideal numbers. Dedekind approaches the topic in a way that is remarkably similar to Frege's discussions in Grundlagen, and as we've already noted, Dedekind is explicit about the Riemannian motives for the particular moves he makes. In particular, Dedekind lays out explicitly that the motivation for his representation-independent presentations is his desire to emulate the methodology incorporated into Riemannian complex analysis.

I'll need some quick background. I'll just wave my hands at crucial points. (Since these developments are exceptionally well covered in the secondary literature, I'm able to restrict attention to just what we need here, with footnote pointers for those who are thirsty for more. $)^{19}$ In the early nineteenth century, a famous fallacious attempt to prove the Fermat theorem failed because the argument assumed falsely that the numbers in a field extending the integers would each have a unique decomposition into prime factors. To give an argument that would compensate for the loss of this property, the first thing that was done was to conceptually split the familiar concept of prime number into two properties that coincide on the integers but not in general: a number $\mathrm{n}$ is irreducible if its only factors are 1 and $\mathrm{n}$ itself, and a number $\mathrm{n}$ is prime if whenever $\mathrm{n}$ divides a product $\mathrm{ab}$ then $\mathrm{n}$ divides $\mathrm{a}$ or $\mathrm{n}$ divides $\mathrm{b}$. Kummer then set out to consider the structures resulting from extending the integers with cyclotomic integers: posited solutions to equations $\mathrm{x}^{\mathrm{n}}=1$ for $\mathrm{n}$ a positive integer. ${ }^{20}$ It would be extremely convenient if cyclotomic integers could be counted on to break down into prime factors. But they can't. Kummer's response can be summed up simply: well, then let's just postulate that there are such factors, and continue as if nothing happened. Of course, it can't be quite that easy: the postulation has to be subject to rules that will keep problems from occurring. The difference between Kummer and Dedekind lies in the shape these rules take: Kummer gives rules that describe how something can be multiplied and manipulated if it is given as an ideal prime factor, while Dedekind actually tells you what these factors are.

${ }^{19}$ Any examination of the topic must begin with the detailed work of Edwards 1975; 1977; 1977a; 1980 and 1987. (This recommendation shouldn't be construed as an acceptance of all of Edwards' methodological obiter dicta concerning the allegedly negligible mathematical power of Dedekind's change of perspective. Ferrierós 1999 has some useful counterbalancing demurrals on various points. He refers to a forthcoming scholarly treatment by Haubrich which appears likely to work as a further counterweight to Edwards' analysis.) Also helpful is Bashmakova and Rudakov 1992, 99-108 and 116-125, especially on the local character of Kummer's method.

${ }^{20}$ Kummer actually explored more general extensions; cyclotomic extensions provide a special case that suffices for our purposes. 
I'll unfold Kummer's approach in connection with a specific example from the cyclotomic integers, though the problems are much more general. ${ }^{21,22}$ Consider the cyclotomic integers resulting from adding the 23 roots of $x^{23}=1 .^{23}$ In this domain, 47 has no prime factors, and so the results that could otherwise be obtained by exploiting the existence of a prime decomposition are unavailable. The general features of these cyclotomic integers tell us to expect 22 distinct ideal prime factors. Not to worry: let's just posit such factors, obtaining an extended system containing the cyclotomic integers and in which 47 does have a decomposition into prime factors. What are these numbers, and why should we believe that there are any? Well, that is a tricky question. As mentioned, Kummer gives rules to tell us how to multiply numbers if they are given as ideal numbers, and he tells us how to see when something given as an ideal number is divisible by another. But we don't actually get a definition. Hence, after introducing Kummer's idea, Dedekind registers this complaint: ${ }^{24}$

... the efforts of Kummer [were] finally rewarded by a truly great and fruitful (féconde) discovery ... [By introducing] ideal prime factors, whose effect is only apparent when they are combined together, he obtained the surprising result that the

${ }^{21} \mathrm{I}$ am brushing by one complication that arises in the general case: the problem of identifying what should count as the integers in the general fields extending the rational numbers. (Edwards 1980 and 1987 puts especially strong emphasis on the mathematical need for a solution to this analytical problem of identifying the properties of integers in general number fields. See also Bashmakova and Rudakov 1992, 106-108) Though I won't be addressing this topic here, but it is worth mentioning as a check against oversimplification, as one more example of the kind of background problem that gave mathematical point to Frege's questions like 'What is a number?' and 'What is the number one?'

${ }^{22}$ I take this example from Edwards 1980, 324-328. Readers who are interested in more specific details (Where did that 47 come from? etc.) can find them there.

${ }^{23}$ The example is not arbitrary. Uniqueness of prime decomposition holds for the cyclotomic integers for $\mathrm{x}^{\lambda}=$ with $\lambda<23$.

${ }^{24}$ Dedekind's characterization of ideal prime factors as having an effect 'that is only apparent when they are combined together' indicates a point of connection to Frege's works that should be noted, though I'm not sure it leads anywhere. The idea that an ideal prime factors is like a radical in chemistry, to be understood in terms of its contribution to compounds, was explicitly stated by Kummer. The remarks were quoted explicitly in Hankel [1867], a book Frege repeatedly cites. This is worth keeping in mind in connection with Frege's ubiquitous chemical metaphors about the 'unsaturatedness' of concepts. (It is especially worth remembering because, as noted in the text, Dedekind's complaint against Kummer is identical to Frege's complaint against 'formalists' at the end of Grundlagen, though Frege presents it as a point about the confusion of concept and object.) Here is a sample of the Kummer remarks quoted by Hankel. (In the sentences surrounding this passage, Hankel mentions other work which attempts to exploit a perceived algebra/chemistry affinity, but none of it caught on.)

Chemical combination corresponds to the multiplication of the complex numbers; the elements, or actually their atomic weights correspond to the prime factors; and the chemical formulae for the decomposition of bodies are exactly the same as those for the decomposition of numbers. We even find the ideal numbers of our theory in chemistry . . . as hypothetical radicals, which have their actuality in compounds (Kummer 1847a, 360, cited in Hankel, 1867, 104). 
laws of divisibility in those numerical domains studied by him were now in complete agreement with those that govern the domain of rational integers.

... Kummer did not define ideal numbers themselves, but only the divisibility of these numbers. If a number $\alpha$ has a certain property $\mathrm{A}$, to the effect that $\alpha$ satisfies one or more congruences, he says that $\alpha$ is divisible by an ideal number corresponding to the property A. While this introduction of new numbers is entirely legitimate, it is nevertheless to be feared at first that the language which speaks of ideal numbers being determined by their products ... may lead to hasty conclusions and incomplete proofs. And in fact this danger is not completely avoided ${ }^{25}$ (Dedekind 1877/ 1996, 57 italics in original).

Some years later, when setting out to provide a finished treatment of this topic, Dedekind reemphasizes the importance of the representation-independent character of an acceptable definition. In this quote, Dedekind has just presented a theory of divisibility of ideals in Kummer style that he regards as not wholly inadequate, but which he nonetheless rejects. He motivates his shift using transparent allusions to Riemannian methods, using the by now familiar catchphrases:

One notices, in fact, that the proofs of the most important propositions depend on the representation of an ideal by the expression $[m a, m(b+\theta)]$ and on the effective realization of multiplication, that is on a calculus ... If we want to treat fields of arbitrary degree in the same way, then we shall run into great difficulties, perhaps insurmountable ones. Even if there were such a theory, based on calculation, it still would not be of the highest degree of perfection, in my opinion. It is preferable, as in the modern theory of [complex] functions to seek proofs based immediately on fundamental characteristics, rather than on calculation, and indeed to construct the theory in such a way that it is able to predict the results of calculation ... Such is the goal I shall pursue in the chapters of this memoir that follow. (Dedekind 1877/ 1996, 102; italics in original, underscoring mine) ${ }^{26}$

In Kummer's treatment, the rules for manipulating an ideal number are inescapably bound to its initial symbolic presentation. Kummer defined ideal numbers by giving an algorithm for computing with them. Dedekind complains that this tells you how to compute - add, divide, multiply - two objects if the two objects are given to you as ideal numbers. But what if two objects are simply given to you? What then? Dedekind's two cited reasons for avoiding the computational option are of interestingly different kinds. On one hand, he indicates a quite tangible methodological reason to prefer the less computational approach: the algorithmic method doesn't generalize to natural cognate cases. (The technique works in the quadratic case, but (so to speak) just barely, and things go crazy in

${ }^{25}$ Dedekind is alluding to the fact that Kummer made some mistakes, and that these mistakes appear to have arisen from Kummer being misled by his mode of expression into assuming more about the numbers he had introduced than he had a right to.

${ }^{26}$ A remark of Hilbert's describing a much later reworking of this material is also worth noting here: 'I have tried to avoid Kummer's elaborate computational machinery so that here too Riemann's principle may be realized and the proofs compelled not by calculations but by thought alone' (Hilbert 1897/1998). 
higher degrees.) The other point goes to the heart of the value of Dedekind's understanding of the Riemann 'conceptual' style, and is once again expressed in familiar clichés: by formulating the problem in the right way we gain a fruitful formulation that will allow us to confidently 'predict the results of computation'. This is not just public relations puffery on Dedekind's part - his objective guides, and is largely attained by the impressive mathematical achievement that these words inaugurate.

So now that we know what Dedekind wants to do and why, how does he do it?

Anyone familiar with Frege's treatment of the Caesar question will have a distinct sense of déjà vu: present an object - the ideal - independently of the representation by taking it to be the set of all the numbers it divides ${ }^{27}$ :

... Since a characteristic property serves to define, not an ideal number, itself, but only the divisibility of the numbers in o by the ideal number, one is naturally led to consider the set $\mathbf{a}$ of all numbers $\alpha$ of the domain $\mathbf{o}$ which are divisible by a particular ideal number. I call such a system an ideal for short, so that to each particular ideal number there corresponds a particular ideal a (Dedekind 1877/1996, 58 italics in original).

Dedekind, like Frege, stresses the importance of fruitfulness as a guide to scientifically important concepts. Note, for example that one of his more famous remarks, taken generally to define his philosophical stance, is followed by remarks that tie the point into the ones we have been discussing, and it invokes a lecture that makes even more explicitly the general point that fruitfulness of novel concepts is a driving mathematical concern:

From just this point of view it appears as something self-evident and not new that every theorem of algebra and higher analysis, no matter how remote, can be expressed as a theorem about natural numbers - a declaration I have heard repeatedly from the lips of Dirichlet. But I see nothing meritorious - and this was just as far from Dirichlet's thought - in actually performing this wearisome circumlocution and insisting on the use and recognition of no other than rational numbers. On the contrary, the greatest and most fruitful advances in mathematics and other sciences have invariably been made by the creation and introduction of new concepts, rendered necessary by the frequent recurrence of complex phenomena which could be controlled by the old notions only with difficulty. On this subject I gave a lecture [Dedekind 1854] ... but this is not the place to go into further detail (Dedekind 1888/1901, 35-36).

${ }^{27}$ Mark Wilson, in his 1999, 248-249 claims that Dedekind supplements his introduction of ideals with a further act of 'erasing' the set-theoretic structure: 'Dedekind always insisted that the "real" factor was obtained from erasing unwanted structure from the set ideal, although this gloss is usually ignored nowadays' (249). This doesn't seem to be true. Wilson presumably has in mind Dedekind's famous appeal to abstraction in his definition of the natural numbers in Dedekind 1888/1901. That seems to be a specific restriction Dedekind places on certain systems of which we have an antecedent conception. Dedekind doesn't do any 'erasing' with ideals in the canonical presentations of the theory like his 1877/1996. (Wilson gives no references for any of his attributions so it is hard to be sure what parts of Dedekind he is referring to.) 
The cited lecture of 1854 - Dedekind's habilitation lecture in front of Gauss and a few others - was devoted to exploring the principles informing the reasonable introduction of new concepts. ${ }^{28}$ In that essay we find remarks reminiscent of the Frege's words concerning fruitfulness as 'the acid test of concepts'. For example, Dedekind remarks - in connection with a hypothetical mineralogist who rejects color as a basis for classification, preferring crystalline structure or chemical composition: 'The introduction of such a concept as a motif for the arrangement of the system is, as it were, a hypothesis which one puts to the inner nature of the science; only in further development does the science answer; the greater or lesser effectiveness of a concept determines its worth or worthlessness' (Dedekind 1854/ 1996, 756). Toward the end of the article, he indicates that the patterns for identification of the most valuable concepts and functions in mathematics are becoming even more complicated, with distinctive novel patterns emerging and continuing to unfold in the theory of elliptic functions (761), presumably referring to the Riemann lectures he was attending at the time. This was prescient; as we've noted, research into elliptic functions (and more generally into functions of a complex variable including the cognate topics of elliptic integrals, Abelian functions, and Abelian integrals) would be at the center of a spectacular reorientation of large areas of mathematics in the coming forty years, within which the broad issues of Dedekind 1854 would be pushed to unexpected depths. Furthermore though to pursue this point would take us far afield - the study of elliptic functions interacted with the study of cyclotomic integers in unexpectedly deep ways. This was shown not just in meta-level philosophical reflection on mathematics but, more importantly, was incorporated within the innovations that drove several streams of ongoing mathematical practice.

There is a further connection between Dedekind's approach to ideals and Frege's rationale for his definition of number that is worth making explicit: in Grundlagen (though not later) Frege makes some critical remarks about 'formalists' that would apply straightforwardly to Kummer's introduction of ideal factors by (as Dedekind puts it) 'characteristic properties'. The point has to be crafted with care because in Grundlagen - though not in [FTA] or Grundgesetze II Frege's use of 'formal' (formal) (which is today tendentiously translated 'formalist') is ambiguous between the view that mathematical objects are signs and a view that we wouldn't call 'formalist' today, that mathematical objects can be introduced through implicit definition by sets of conditions. In later writings,

${ }^{28}$ Frege most likely never saw Dedekind 1854 , as it remained unpublished until volume III of Dedekind's collected works appeared in 1930. It's not inconceivable that Frege might have somehow obtained a copy of the lecture but lacking any evidence to this effect, the default assumption is surely that he didn't. Frege did read Dedekind 1888, and in fact devoted a session of his mathematics seminar to studying it; but it came out too late to influence anything in Grundlagen of 1884. Dedekind 1877 appeared in French; it is however, a variation on Dedekind 1879, which Frege would have had easy access to and almost surely did read. 
'formalist' is an appropriate translation, as 'formal' is used more narrowly to mean only the view that mathematical objects are signs. ${ }^{29}$ It is important to be clear on this, as the remarks about 'formal' theories in Grundlagen are often misread by projecting later discussions back on to them.

Frege connects his critique of 'formal theories' to one of his 'three fundamental principles': 'never to lose sight of the distinction between concept and object' ([FA]):

As to the [distinguish concept and object] point, it is a mere illusion to suppose that a concept can be made an object without altering it. From this it follows that a widely held formal theory of fractional, negative, etc. numbers is untenable ([FA]) (emphasis mine).

Toward the end of Grundlagen Frege revisits the point in a critical discussion of Hankel (\$92-\$99). He notes that some sets of conditions cannot be satisfied, and so to assume they can be satisfied would generate a false result. Again, the concept - object distinction is invoked:

This is the error that infects the formal theory of fractions and of negative and complex numbers [Footnote: Cantor's infinite numbers are in like case.] It is postulated that the well-known rules of calculation shall still hold, where possible, for the newly introduced numbers, and from these their general properties and relations are deduced. If no contradiction is anywhere encountered, the introduction of the new numbers is held to be justified, as though it were impossible for a contradiction still to be lurking somewhere nontheless, and as though freedom from contradiction amounted straightaway to existence.

$\S 97$ That this mistake is so easily made is due, of course, to the failure to distinguish concepts and objects ([FA] 108).

Frege's arguments in this section are completely general: they apply to any effort to introduce objects just by listing conditions, not just those which involve the stance that mathematical objects are signs. Though Frege doesn't mention Kummer or ideal factors here, reference to them is presumably included in the broad sweep of his discussion of theories of complex numbers. Indeed, in Grundgesetze II (\$144) and the essay on Schubert's numbers ([SN] 264), Frege uses the introduction of primitive roots as illustrations in arguments that are identical to the Grundlagen arguments under discussion here. (Frege notes archly in [SN] that when Gauss addressed this kind of question in connection with the Gaussian

${ }^{29}$ In subsequent writings [FTA] and Grundgesetze II, 'formal' is applied consistently and exclusively to the 'mathematical objects are signs' view. One tangible reflection of Frege's changed usage is that in Grundlagen Frege counts Hankel's work and Cantor's introduction of infinite numbers as examples of 'formal theories' (formale Theorien). In Grundgesetze II (§ 145) he explicitly distinguishes uses of 'formal', and states that Cantor is not a formalist in the 'mathematical objects are signs' sense, adding that Hankel is an unclear case because he didn't clearly distinguish sign and signified. To reflect this, I am translating "formal" in Grundlagen with the neutral "formal" rather than 'formalist', which is today loaded with overtones of the 'numbers are signs' view. 
integers he proved the existence of the primitive roots he posited.) This suggests strongly that pre-Dedekindian introductions of ideal factors are among those definitions that are counted as confusing concept and object. And though Frege doesn't mention it, Dedekind's definition of ideals was one that did get things right as far as the concept-object distinction is concerned.

We should take stock here of just what Frege's contribution was in all this. The idea that objects should be introduced in a representation-independent way and that this imperative arose in part from a fact about the way that mathematical reasoning proves most fruitful was already in place in Dedekind's rigorous application of Riemann's methods in connection with generalized numbers. The idea that the ideal method demands global as opposed to local definitions was also in the air in Frege's environment. Since these were traceable back to the principles informing Riemann's complex analysis (and its prehistory in Gauss' musings), there was a compelling rationale available for the claim that these were methodological principles that would support fruitful research.

Of course, it is one thing to distinguish informally, as Dedekind does, between 'functions given by inner characteristics' and the subsequently derived features of 'external forms of representation'. It is another to make clear sense of what this sort of thing means. Frege's distinctive contribution was to produce a deep logical analysis and philosophical rationale for this Riemannian perspective. Say it is granted that a powerful mathematical advantage is gained by introducing the object of study as a 'self-subsistent' object rather than as tied to some representation. It isn't unreasonable to ask just what it means to say you've introduced something in a representation independent way. What is the logical structure underlying such a transition? If we are to take a primitive concept of function and rely extensively on non-constructive function existence arguments, someone has to spell out what the logic of function existence arguments is. And just when is an object introduced in a representation-independent way? What principles - logical or otherwise - govern the successful, representation-independent introduction of an object? It is possible to debate just how much was ultimately added to the mathematical situation by specific details of Frege's logical analysis. I will leave this question for elsewhere. But we should be clear, when evaluating Frege's remarks on the general methodological objectives of his work, that he can be taken to be laying out the logical basis of a true mathematical revolution whose nature had not yet been completely understood, and would not be fully understood for many years to come.

\section{Appendix: Broad and narrow definitions revisited: Did Frege change horses in Midstream?}

Another example that illustrates the Riemannian preference for broader rather than narrower definitions is best considered in an appendix, since it also allows us to 
address a separate puzzle. When Frege laid out the definition with Hume's principle, he appears to have done everything he needed for the subsequent development of arithmetic in Grundgesetze. The subsequent definition, crafted to address the Caesar problem, seems mathematically idle. In Frege's formal derivations the 'official' definition is used only to derive the Hume's principle definition Frege set aside, and the Hume's principle definition does all the subsequent work. It has even been suggested (as we'll see in a moment) that Frege simply changed his mind while writing Grundlagen and the shift from one definition to another reflects this. This example will help ease the sense of oddness that prompts such suggestions. In Riemann's treatment of the zeta function we'll find a broad definition given in a case where an already familiar narrow definition seems to do most of the work. (It isn't so broad as to decide the value of the zeta function on Julius Caesar, of course, but even on a less than maximally global scale it illustrates the pattern we need.)

There is a crucial difference in the Riemann and Weierstrass approaches in connection with definitions. Weierstrass' style of definition was local and piecemeal while, as noted, Riemann sought simple, global definitions that allow us to exploit global properties. ${ }^{30}$ Weierstrass' approach was to some extent a straightforward consequence of his techniques of 'arithmetization' and the correlated attitude that 'the ultimate aim is the representation of a function'. To obtain a function over the entire complex plane, the function is often patched together through analytic continuations: a sequence of functions defined on a chain of discs, with each local function defined on the corresponding disc by a complex power series converging on that disc. ${ }^{31}$

In the watershed paper Riemann 1859/1974 Riemann attacks the question of the distribution of prime numbers by first giving a global definition of the $\zeta$ function. This function was well-known to be relevant to the distribution of primes, but the function had only a partial definition. It was given by a series:

${ }^{30}$ I am indebted to Edwards 1974, 9-13 and passim for drawing my attention to this feature of Riemann's approach and to the contrast with Weierstrass. Edwards sums up the relevant methodological contrast succinctly:

... Riemann does not speak of the 'analytic continuation' of the function $\Sigma_{i=1}^{\infty} 1 / n^{\mathrm{s}}$ beyond the halfplane Re $s>1$ but speaks rather of finding a formula for it which 'remains valid for all s'. This indicates that he [sought a single global extension rather than] a piece - by - piece extension of the function in the manner that analytic continuation is customarily taught today. The view of analytic continuation in terms of chains of discs and power series convergent in each disc descends from Weierstrass and is quite antithetical to Riemann's basic philosophy that analytic functions should be dealt with globally, not locally in terms of power series (Edwards 1974, 9).

${ }^{31}$ Though analytic continuation is most commonly associated with Weierstrass because of the importance it played in his general approach, the fact of the uniqueness of analytic continuations was known to Riemann too, and he used the fact when it was helpful. The point is taken up in Neuenschwander 1980. 
$\zeta(\mathrm{s})=\sum_{\mathrm{i}=1}^{\infty} 1 / \mathrm{n}^{\mathrm{s}}$, which converges for complex numbers with real part greater than 1 and diverges elsewhere. A key equivalence, known to Euler (the 'Euler product formula'), also holds only in this restricted domain:

$$
\begin{aligned}
& \zeta(\mathrm{s})=\Sigma^{\infty}{ }_{\mathrm{i}=1} 1 / \mathrm{n}^{\mathrm{s}}=\Pi_{\mathrm{p}} 1 /\left(1-1 \mathrm{p}^{\mathrm{s}}\right) \\
& \text { (with the product taken over all primes } \mathrm{p} \text { ). }
\end{aligned}
$$

From the first paragraph, Riemann 1859 shows itself to be engaged, in practice, with themes to which Frege later gave theoretical expression. Riemann explicitly crafts his definition of the zeta - function with an eye to ensure the definition was 'always valid' (immer gültig) over the entire complex plane (except at one pole where it is known to be undefined), eschewing repeated analytic continuations via power series in favor of a single, global definition that settles all (but one) of the complex cases at a stroke:

$$
\zeta(v)=\int_{-\infty}^{\infty} \frac{(-1)^{v} \mathrm{dx}}{\mathrm{x}\left(\mathrm{e}^{\mathrm{x}}-1\right)}
$$

The product formula is a special case, restricted to the domain in which the series $\Sigma^{\infty}{ }_{i=1} 1 / n^{i}$ converges. To appreciate what is at stake here, it is important to recognize that it is possible to continue to do analytic number theory just in terms of the product formula, leaving the global definition aside. ${ }^{32}$ It was not clear at the beginning just how much of a difference, if any, the global definition would make. ${ }^{33}$ Neither in Riemann's original paper nor in any subsequent discussions was the desire for a general definition seen as incompatible with the idea that at least for a large range of key questions, the restricted definition captured the essential heart of the general definition.

Of course, there is a difference of degree in the Frege and Riemann approaches: Frege's concern with full determinacy of defined expressions (Julius Caesar, the moon, ... .) - and his conception of this as bound up with the possibility of formulating and applying logical laws - exceeds anything I have found in Riemann. Riemann was not at all concerned with the values of functions on arguments like 'Julius Caesar' or 'the moon'. But Frege's approach can be seen as a more general version of Riemann's, that has Riemann's as a special case. The crucial points here are these: a) The emphasis Frege places on total definition of

${ }^{32}$ To locate the point in Frege's environment, consider the habilitation thesis of Frege's Jena colleague Adolf Piltz, (Piltz 1884) addressed the distribution of primes using the zeta function, and almost all the work in the thesis was done by one of the series forms or the Euler product formula. Riemann 1859 is mentioned approvingly, but the global definition of the zeta function is absent.

${ }^{33}$ For what it is worth, this opinion is still espoused, for example in Patterson 1988, 1) who remarks that the Euler product formula is 'the reason for the importance of the zetafunction'. 
functions and the avoidance of piecemeal procedures of definition was not mathematically inert. It would have favored one mode of proceeding - Riemann's over another - Weierstrass' - in work that was sufficiently renowned, particularly in Göttingen - that Frege was certain to be familiar with it; b) The position that would result from adopting Frege's strictures stands in opposition to the procedure exploited in Weierstrass' 'arithmetization of analysis'. ${ }^{34}$ c) Finally, the affinity between Riemann's innovation and Frege's definition of number appears against the background of more broadly philosophical similarities concerning the value of mathematical innovations, particularly as this aspect of Riemann's approach is interpreted by Dedekind.

This helps to set several otherwise puzzling features of Frege's practice in a different light. As noted, it has been taken to be a puzzle why Frege should bother with the Caesar detour at all, given that from the point of view of the logical work he wants to do, it seems that everything he needs is given by the Hume's principle definition he considers and rejects. The theorems of arithmetic derived in Grundgesetze could have been derived from the system resulting from replacing basic law V with Hume's principle. Frege couldn't have failed to be aware of this, since his only essential application, in Grundgesetze, of his final, 'official' definition of number is to use it to prove the Hume principle, which then does nearly all of the work. Some commentators have even posited that Frege changed his mind abruptly in the middle of composing the book. ('[P]ulled a switcheroo without adequately informing his readers of the shift' in the words of Wilson 1999, $258)^{35}$ Wilson proposes a speculative reconstruction of an overall change of mind that might have prompted the perceived shift. Setting aside the question of the likelihood of the reconstruction (though I do find it unconvincing), I don't think there is anything that needs to be explained. Frege first spells out the equivalence that does much of the subsequent work, but which is not defined in general, and then gives a definition which has full generality. This is just what Riemann did in observing the importance of the series representation of the zeta-function, and especially of the Euler product formula, despite the fact that these two functions are defined only on complex numbers whose real part is greater than one. He gives a more general definition (undefined only at one point), much of whose main work is still done by the restricted parts. (Similar things could be said of the Dedekind definition of ideals in relation to Kummer's algorithms.) So I don't see that Frege or his audience would have held there to be anything at all out of the ordinary about Frege's double definition approach in Grundlagen. As high-profile examples

${ }^{34}$ Note that here too a practical issue runs parallel with the theoretical one. In this particular case of the zeta-function the power series representation turns out to be useless in practice (according to Laugwitz 1999, 84).

${ }^{35}$ See also Sluga 1980, 127 who makes a similar suggestion (though without the imputation of dishonesty to Frege). 
around Frege exemplified, sometimes it just turns out that you need one 'official' definition to be global and another, restricted one to haul the water.

\section{REFERENCES}

Bashmakova, I. and Rudakov, A. 1992, "Algebra and Algebraic Number Theory," in: A. Kolmogorov and A. Youshkevich, eds., A. Shenitzer trans., Mathematics of the Nineteenth Century, Boston: Birkhäuser.

Benacerraf, P. 1981, "Frege: The Last Logicist," in: P. French, T. Uehling and H. Wettstein, eds., Midwest Studies in Philosophy vol. 6, Minneapolis: University of Minnesota Press.

DeDEKIND, R.1854/1996, "On the Introduction of New Functions in Mathematics," W. Ewald (trans. 1996), pp. 754-762 (delivered June 1854), German original in Dedekind's Werke III p. 428-438.

DedeKIND, R. 1871, Supplement X to Dirichlet.

Dedekind, R. 1872/1901, “Continuity and Irrational Numbers," W. Behman, trans., Chicago: Open Court (original pub. 1872, translated 1901, page reference to Dover reprint "Essays on the Theory of Numbers" of 1963).

DedeKind, R. 1876a, “Letter to R. Lipschitz,” (Dedekind's Werke III, pp. 464-482).

DeDEKIND, R. 1877, "Schreiben an Herrn Borchardt über die Theorie der elliptischen Modulfunctionen," Journal für Reine und Angewandte Mathematik 83 pp. 265-292 (Dedekind's Werke I pp. 174-201).

Dedekind, R. 1877/1996, Theory of Algebraic Integers, J. Stillwell, trans., Cambridge: Cambridge University Press (original published 1877 as Sur la Théorie des Nombres Entières Algébraiques, Paris: Gauthier - Villars).

DedeKIND, R. 1879, Supplement XI to Dirichlet.

DedeKind, R. 1880, "Letter to H. Weber," (Dedekind's Werke III, p. 488)

DeDEKIND, R. 1888/1901, “The Nature and Meaning of Numbers," W. Behman (trans.) Chicago: Open Court (original published 1888, translated 1901, page reference to Dover reprint "Essays on the Theory of Numbers" of 1963).

Dedekind, R. 1895, "Über die Begründung der Idealentheorie," Nachrichten von der Königlichen Gesellschaft der Wissenschaften zu Göttingen Mathem. - Phys. Klasse. pp. 106-113 (page references to Dedekind's Werke vol. II pp. 50-58).

Dedekind, R. and Weber H. 1882, "Theorie der algebraischen Funktionen einer Veränderlichen," Journal für Reine und Angewandte Mathematik 92, pp. 181-290 (dated 1880), reprinted in Dedekind's Werke I pp. 238-349.

Edwards, H. 1974, Riemann's Zeta Function, New York: Academic Press.

Edwards, H. 1975, "The Background of Kummer's Proof of Fermat's Last Theorem for Regular Primes," Archive for History of Exact Sciences 14, pp. 219-236.

EDWARDS, H. 1977, Fermat's Last Theorem, New York: Springer.

Edwards, H. 1977a, "Postscript to 'The Background of Kummer's Proof . ..,", Archive for History of Exact Sciences 17, pp. 219-236.

EDWARDS, H. 1980, “The Genesis of Ideal Theory,” Archive for History of Exact Sciences 23, pp. 321378.

Edwards, H. 1987, "Dedekind's Invention of Ideals," in: E. Phillips, ed., Studies in the History of Mathematics, MAA monographs 26, pp. 8-20.

FERRIERós J. 1999, Labyrinth of Thought: A History of Set Theory and its Role in Modern Mathematics, Boston: Birkhäuser.

Key to Frege references in text:

[BLC] "Boole's Logical Calculus and the Concept-Script," in Posthumous Writings.

[FA] 1884, The Foundations of Arithmetic, J. L. Austin ed. and trans. 2nd rev. edn., Evanston: Northwestern University Press.

[FTA] 1885, "Formal Theories of Arithmetic," in Collected Papers.

[GzII] 1903, Grundgesetze der Arithmetik vol II, Jena: H. Pohle. 
[MCE] 1874, "Methods of Calculation Based on an Extension of the Concept of Quantity," in Collected Papers.

[SN] 1899, "On Mr. Schubert"s Numbers," in Collected Papers.

Frege G. 1979, Posthumous Writings, H. Hermes, F. Kambartel and F. Kaulbach, eds., Oxford: Basil Blackwell.

Frege, G. 1984, Collected Papers on Mathematics, Logic and Philosophy, B. McGuinness, ed., Oxford: Basil Blackwell.

Gray, J. 1994, "On the History of the Riemann Mapping Theorem" Rendiconti del Circolo Mathematico di Palermo supplement 34, pp. 47-94.

Gronau, D. 1997, "Gottlob Frege, A Pioneer in Iteration Theory," in Iteration Theory (ECIT 94) Proceedings of the European Conference on Iteration Theory, Opava Grazer mathematische Berichte \#334, pp. 105-119.

Hankel, H. 1867, Vorlesungen über die Complexen Zahlen und Ihren Functionen vol. I Theorie der Complexen Zahlensysteme, Leipzig: Teubner.

Hilbert, D. 1897/1998, The Theory of Algebraic Number Fields, I. Adamson, trans., (originally published 1897) Berlin: Springer Verlag.

Husserl, E. 1891, Philosophie der Arithmetik. Psychologische und Logische Untersuchungen vol 1, Halle: C. Pfeffer.

Kummer, E. 1847, “Zur Theorie der Complexen Zahlen,” Monatsberichte Akad. Wissenschaft Berlin, pp. 87-96 (also Crelle's Journal 1847 and Collected Papers pp. 203-210) page references to English translation by T. Cope in Smith 1959, pp. 119-126.

Kummer, E. 1847a, "Über die Zerlegung der aus Wurzeln der Einheit gebildeten complexen Zahlen in ihre Primfactoren," Journal für Reine und Angewandte Mathematik 35, pp. 327-367(Collected Papers pp. 211-251).

Laugwitz, D. 1992, “ “Das letzte Ziel ist immer die Darstellung einer Funktion,': Grundlagen der Analysis bei Weierstraß 1886 - historische Wurzeln und Parallelen," Historia Mathematica 19, pp. 341-355.

Laugwitz, D. 1999, Bernhard Riemann: 1826-1866 Turning Points in the Conception of Mathematics, A. Schnitzer, trans., Boston: Birkhäuser.

Liebmann, H. 1918, "Die Angenäherte Ermittelung harmonischer Funktionen und konformer Abbildungen," Sitzungsberichte Bayerische Akad. Wissenschaften Math.-Phys. Klasse pp. 385416.

NeuenschWANDER, E. 1980, "Riemann und das 'Weierstrasssche Prinzip' der analytischen Fortsetzung durch Potenzreihen," Jahresbericht der Deutschen Mathematiker-Vereinigung 82, pp. 1-11.

Patterson, S. 1988, An Introduction to the Theory of the Reimann Zeta-Function, Cambridge: Cambridge University Press.

PIltz, A. 1884, Über die Häufigkeit der Primzahlen in Arithmetischen Progressionen und über Verwandte Gesetze, Jena: Venia Docenti Thesis.

Remmert, R. 1991, Theory of Complex Functions, R. Burkel, trans., Berlin: Springer Verlag.

Riemann, B. 1859/1974, "On the Number of Primes Less than a Given Magnitude," H. Edwards, trans., published as an appendix to Edwards 1974, pp. 299-310.

Siegel, C. 1969, Topics in Complex Function Theory vol. 1 Elliptic Functions and Uniformization Theory A. Shenitzer, trans., New York: Wiley Interscience.

SlugA, H. 1980, Gottlob Frege, London: Routledge and Kegan Paul.

Smith, D. 1959, A Source Book in Mathematics, Mineola NY: Douer.

Stahl, H. 1899, Elliptische Functionen. Vorlesungen von Bernhard Riemann, Leipzig: Teubner.

TAppenden, J. 1995, "Geometry and Generality in Frege's Philosophy of Arithmetic," Synthèse 102, pp. 319-361.

Tappenden, J. 1995a, "Extending Knowledge and 'Fruitful Concepts': Fregean Themes in the Foundations of Mathematics," Noûs 29, pp. 427-467.

TAPPEndEN, J. forthcoming a, "The Riemannian Background to Frege's Philosophy," in: J. Ferreirós \& J. J. Gray, eds., The Architecture of Modern Mathematics: Essays in History and Philosophy, Oxford: Oxford University Press. 
Tappenden, J. forthcoming b, Philosophy and the Origins of Contemporary Mathematics: Frege's Mathematical Context, Oxford: Oxford University Press.

TAPPENDEN, J. in preparation c, "Reflections on Mathematical Explanation (Why do Elliptic Functions have Two Periods?)".

Walsh, J. 1973, "History of the Riemann Mapping Theorem," American Mathematical Monthly 80, pp. 270-6.

WeIERSTRAss, K. 1886/1988, Ausgewählte Kapitel aus der Funktionenlehre. Vorlesung, gehalten in Berlin. Mit der akademischen Antrittsrede, Berlin 1857 und drei weiterenn Originalarbeiten von K. Weierstrass aus den Jahren 1870 bis 1880/6 edited with commentary by R. Siegmund-Schultze Teubner - Archiv zur Mathematik, Bd. 9, Liepzig: Teubner.

Weiner, J. 2000, Frege, Oxford: Oxford University Press.

Wilson, M. 1999, "To Err is Humean," Philosophia Mathematica 3, 7, p. 249. 